Results There were a significant difference in the levels of HDL $(1,2 \pm 0,31 \mathrm{mmmol} / 1$ to $69 \pm 0,01 \mathrm{mmol} / \mathrm{l}, \mathrm{p}<0,001)$. In these children were indicated increase level of total cholesterol by LDL fraction to $2,35 \pm 0,18 \mathrm{mmol} / \mathrm{l}$. IA was in 2.97 times higher in patients with mountainous region to according the control group (2.67 and 0.90). Changes in hemostasiogramme were identificated in 54\% children (an increase of activated recalcification time $(74,76 \pm 5,06 \mathrm{~s}$ and $64,76 \pm 2,04 \mathrm{~s}, \mathrm{p}<0.05)$, an increase of concentration of fibrinogen $(17,53 \pm 1,63 \mathrm{~s}$ and 11,32 \pm $0,77 \mathrm{~s}, \mathrm{p}<0.001)$. According to our research were higher levels of Antiphospholipid IgM, than $\operatorname{IgG}(2,73 \pm 0,34$ to $2,03 \pm$ $0,24, \mathrm{U} / \mathrm{ml}, \mathrm{p}<0.02$ ), which varies within the reference values, but have different signs with dates of the control group.

Conclusion These dates presented the risk of thrombogenesis, but non significant. The levels of IL- 1 and IL -6 were in the range of control values, but have a tendency to decline, according to our data. This fact indicated about the decrease in production of interleukins of child's organism in the mountains region.

\section{PO-0032 URINARY TRACT INFECTION IN CHILDREN AFTER CARDIAC SURGERY: INCIDENCE, RISK FACTORS AND OUTCOME}

M Kabbani, A Fatima, R Singh, R Shafi, J Idris, A Mehmood, H Al Muhaidib, S Ismail, 0 Hijazi. Cardiac Sciences, King Abdulaziz Medical City, Riyadh, Saudi Arabia

10.1136/archdischild-2014-307384.709

Introduction Urinary tract infection (UTI) can prolong hospitalisation, and increase morbidity. Catheter associated UTI (CAUTI) is a major cause of UTI.

Objective To determine the incidence, risk factors, aetiology and outcome of UTI in postoperative cardiac children.

Patients and methods This is retrospective cohort study. All post-operative patients admitted to PCICU during 2012 were included. Patients were divided into: group (1) Patients who developed and group (2) patients who did not develop UTI. The two groups were compared for demographic and other variable predictors for UTI.

Results 413 post-cardiac surgical children were included. Group (1) had 29 patients (7\%) all had CAUTI. Foley catheter utilisation ratio was 44\%. CAUTI density rate was 18 per 1000 catheter days. Logistic regression analysis demonstrated that risk factors for developing UTI were: duration of Foley $(p<0.002)$, associated syndrome $(\mathrm{p}=0.01)$ and prolonged PCICU and hospital stay $(\mathrm{p}<0.05)$. Gram-negative were responsible for $63 \%$ and Candida for (24\%) of the CAUTI. ESBL caused 30\% and MDRO caused $10 \%$ of our patients CAUTI.

Conclusion Foley catheter duration, presence of syndrome and prolonged PCICU and hospital stay were the main risk factors for CAUTI in postoperative paediatric cardiac patients. Resistant Gram-negative were the main cause for CAUTI with one third of CAUTI cases caused by MDRO or ESBL organisms. The cases with CAUTI were generally sicker and with more morbidity. The study will establish a baseline clinical indicator for monitoring quality improvement and the future measures to minimise CAUTI incidence, and its co-morbidity.

\section{PO-0033 REFERRALS FOR ECHOCARDIOGRAMS IN A DISTRICT GENERAL HOSPITAL - ARE WE ON THE PULSE?}

KL Kok, K Sandhu. Paediatrics, Princess Alexandra Hospital, Harlow, UK
Background and aims Echocardiograms are a major resource for diagnosing congenital and acquired heart disease. Paediatricians with special expertise in Paediatric Cardiology are now at the forefront of the paediatric cardiac care service. The aim of this audit was to report on the quality of referrals - investigating the indications for referral, documentation of symptoms and signs.

Methods The medical notes of 46 children who attended outpatient echocardiogram clinic were retrospectively reviewed (April to June 2012). We looked at indications for referral, documentation of symptoms and signs in the initial referral letters.

Results 41 out of 46 children attended the clinics, of which 39 sets of notes were available to be reviewed. 22 were new and 17 were follow-ups. The main indications for referral were presence of murmur, family history, congenital heart disease, syndromes and Kawasaki Disease.

16 children were referred because of murmur, 12 of whom were asymptomatic. Half did not comment on grade, position or radiation of the murmur. One-third did not have documentation of femoral pulses, signs of heart failure (crackles on auscultation, hepatomegaly, oedema).

Conclusions Indications for referral to echocardiogram clinic were appropriate. However, the quality of documentation of basic examination findings was poor. This audit starts a discussion regarding setting the standard for quality of referrals to echocardiogram clinic, and highlights deficiencies in documentation. Recommendations include a standardised pro forma and an emphasis during general practice study days.

\section{PO-0034 THE INCREASED SERUM LEVELS OF INTERLEUKIN-21 IN KAWASAKI DISEASE}

H Lee. Pediatrics, Yonsei University Wonju College of Medicine, Wonju, Korea

\subsection{6/archdischild-2014-307384.711}

Purpose It has been reported that serum level of immunoglobulin $\mathrm{E}(\mathrm{IgE})$ is increased in patients with Kawasaki disease (KD) after acute phase. However the exact mechanism of increasing IgE is yet to be revealed. We investigated whether the interleukin-21 (IL-21) could be related with the high IgE in KD. Instead of IL-4, IL-21 was focused in this study because it has been reported that its level is increased in various autoimmune vasculitis.

Methods From June 2008 to June 2010, 49 patients with KD admitted in Wonju Christian Hospital and 13 controls with high fever due to unknown infection who had no history of KD were included in this study. The sera from patients and controls were collected and checked in terms of immunoglobulin E (Chemiluminescent method, Siemens, Munich, Germany) and IL-21 (ELISA, eBioscience, San Diego, USA).

Results The median age of patients with KD was 3 years of age (range: $0.4-10)$ and that of controls was 7 years of age (range: 1-12). The group of patients with KD was composed of 39 complete $\mathrm{KD}$ and 10 incomplete KD. Among patients with KD, 10 patients had coronary arterial dilatation (CAD) and 39 patients had no coronary complications. The median value of IL-21 in patients with KD was significantly increased as $466 \mathrm{pg} / \mathrm{mL}$ (range: 0-1544) while that value in controls was $<62.5 \mathrm{pg} / \mathrm{mL}$ (range: $0-825 \mathrm{pg} / \mathrm{mL})(\mathrm{p}<0.01)$. We could not find the significant correlation between the serum level of IgE and that of IL21 in patients with $\mathrm{KD}$ (Spearman $\mathrm{R}=0.2, \mathrm{p}=0.08$ ) though $30 \%$ of patients with KD showed increased IgE more than 100 $\mathrm{IU} / \mathrm{mL}$. In addition, our data showed no significant difference 